\title{
Best Paper Award in Memory of Jacques Calmet
}

(c) Springer-Verlag GmbH Germany, part of Springer Nature 2021

The 2020 AAECC Best Paper Award in Memory of Jacques Calmet is awarded to

Curtis Bright,

Kevin Cheung,

Brett Stevens,

Dominique Roy,

Ilias Kotsireas,

Vijay Ganesh

for their paper

"A nonexistence certificate for projective planes of order ten with weight 15 codewords"

published in Applicable Algebra in Engineering, Communication and Computing 31 (2020), no. 3-4, 195-213.

The prize is awarded for an innovative new approach in studying the important classical problem of existence of finite projective planes. Using techniques from the fields of symbolic computation and satisfiability checking, the authors verify one of the cases used in the famous result proved in 1973 that projective planes of order ten do not exist. In particular, the authors show that there exist no projective planes of order ten that generate codewords of weight fifteen, a result originally shown via an exhaustive computer search, by providing a simple satisfiability (SAT) instance and a certificate of unsatisfiability that can be used to automatically verify this result.

The editorial board wishes to extend its warmest congratulations to the winners! 